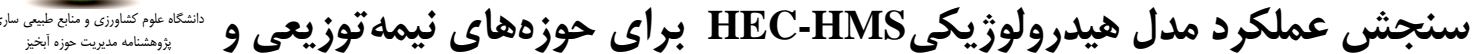 يكيار جه (مطالعه موردى: حوزهيد آبخيز كوهسوخته)
}

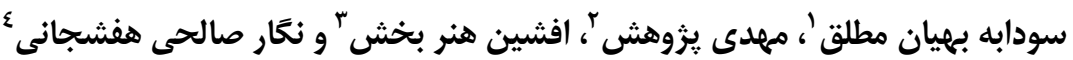

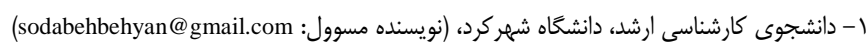

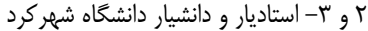

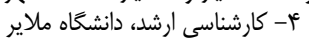

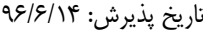

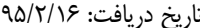

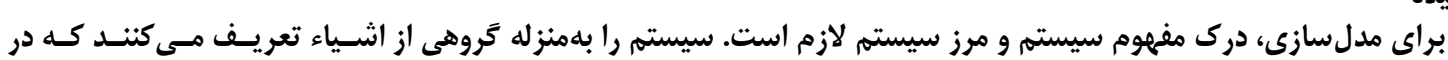

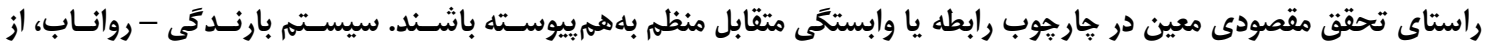

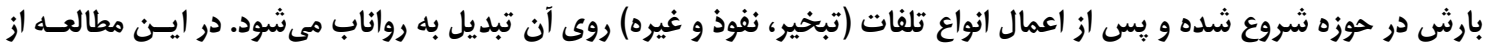

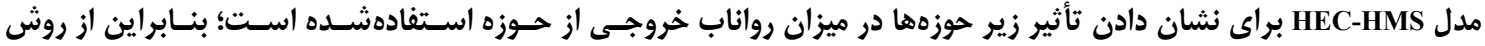

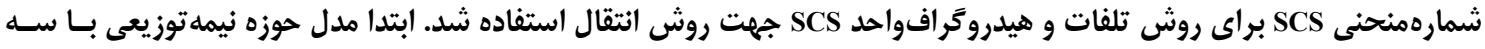

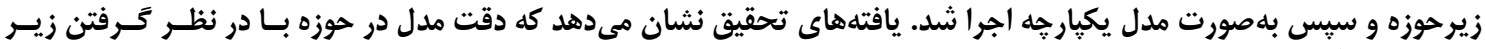

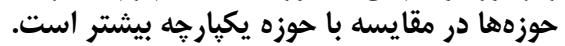

\section{وازههاى كليدى: حوزه يكيارجه، حوزه نيملتوزيعى، مدل هيدرولوزيكى، SCS}

محاسبه مىشود. اين مدل مى تواند در مناطق فاقـد آمـار و در

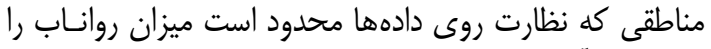

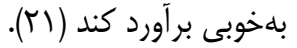

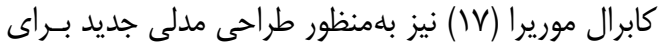

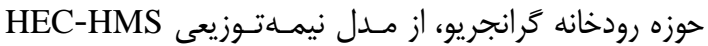

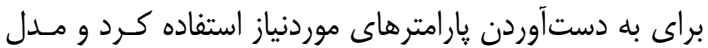

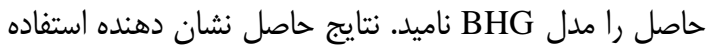

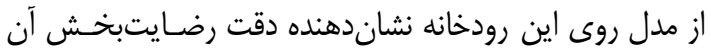
در برآورد ورودى موى ائ رود مدل HEC-HMS بودانه نشان

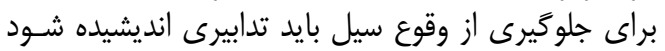

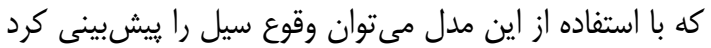

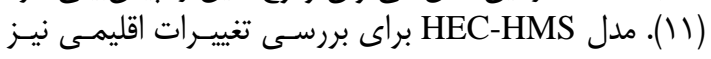

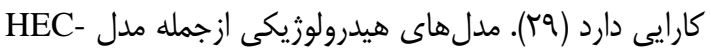

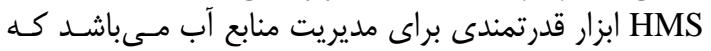

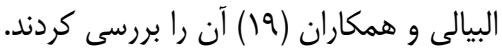

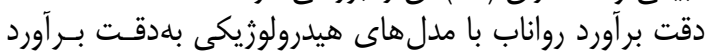

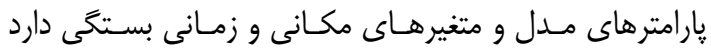

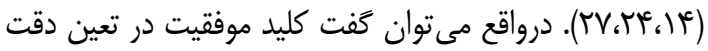

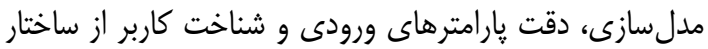

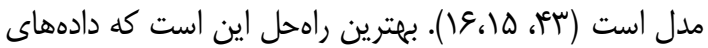

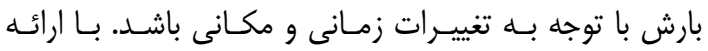

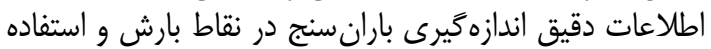

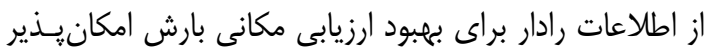

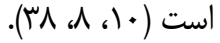
مــــ-HMS

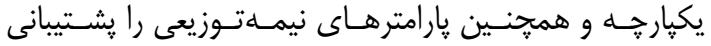

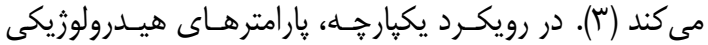

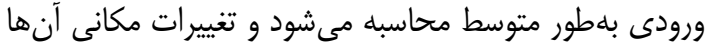
مدنظر نيست. در تحقيقى كه اسدى و بوستانى (ع) بلمنظور بررسـى كـاربرد

5- Pre-and-Post Processor for HEC-HMS, 2004
مقدمه

شبيهسازى تقليدى است از عملكــرد فرآينـد يـا سيسـتهم

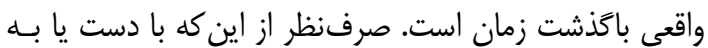

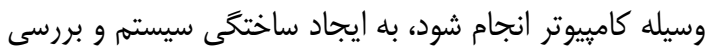

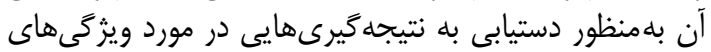

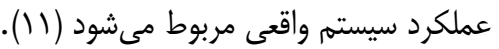

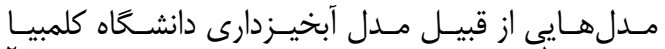

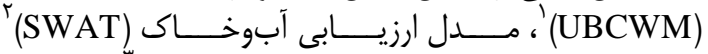

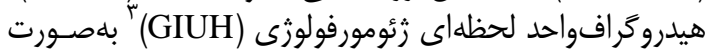

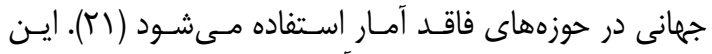

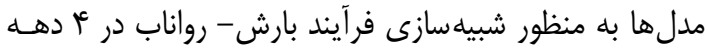

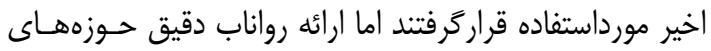

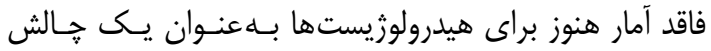
باقى مانده است (• مأم).

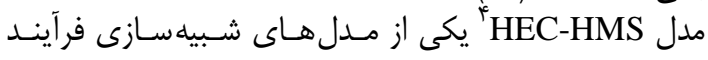

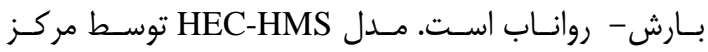

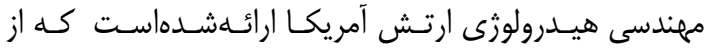

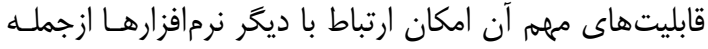

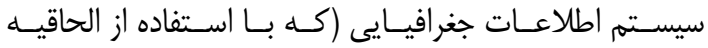
HEC-GEO HMS HEC-GEO HMS بسيارى از دادههاى موردنياز را كـه نيـاز

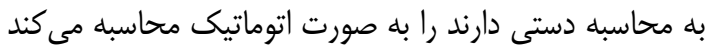

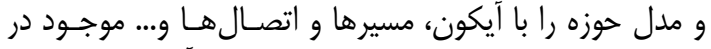

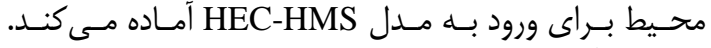

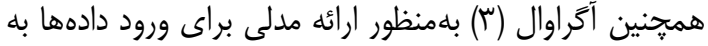

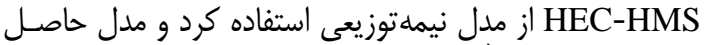
ناميد. ${ }^{\circ}$ Per-Pro2004 I, در مدل HEC-HMS براى محاسبـه روش تلفـات، انتقـال و

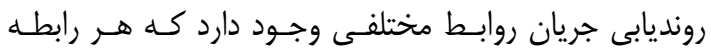

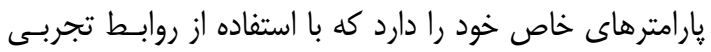




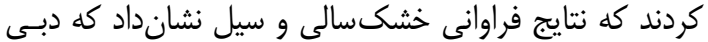

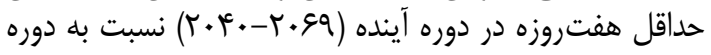

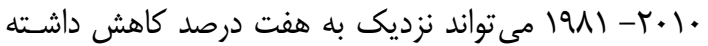

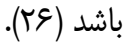

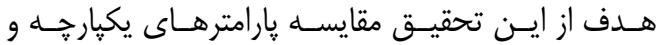

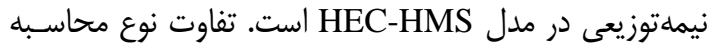

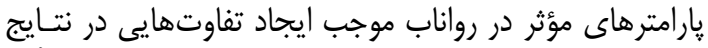

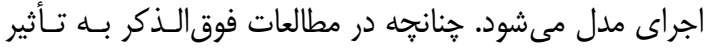

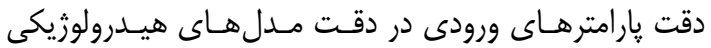

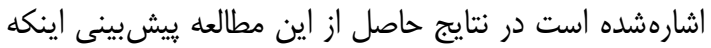

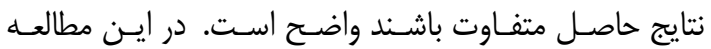

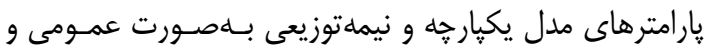

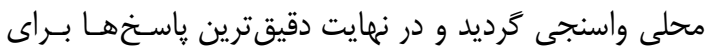

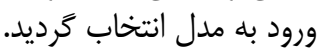

\section{مواد و روشها موزها} مشخصات حوزه مورد مطالعه

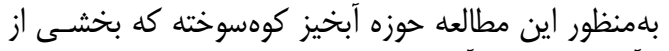

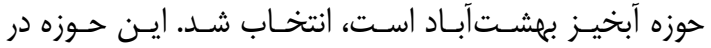

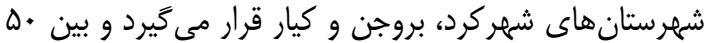

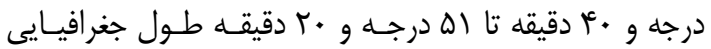

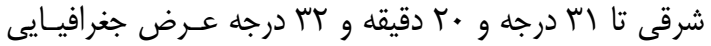

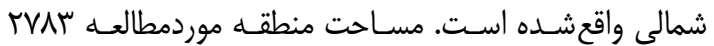

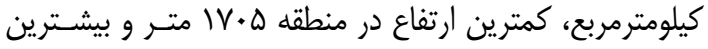

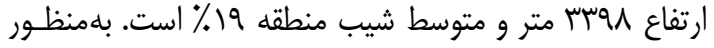

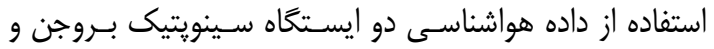

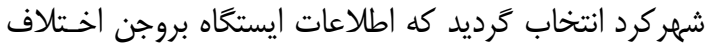

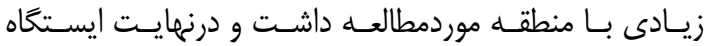

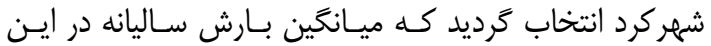

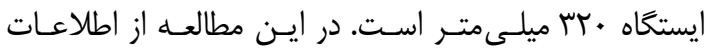
هيدرومترى ايستخاه هيدرومترى كوهسوخته استفاده كرديد.
مدل HEC-HMS انجام دادند، نشان داد نتايج بهدستآمده از مدل نيمهتوزيعى دقت بيشترى دارد.

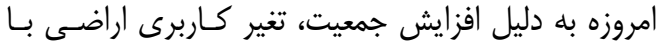

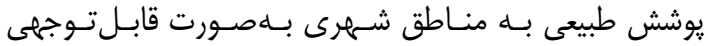

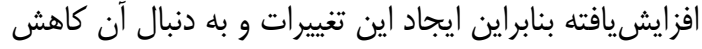

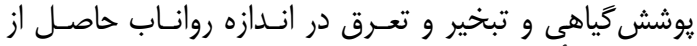

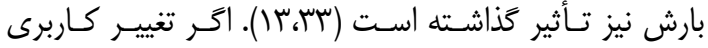

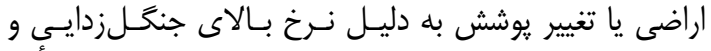

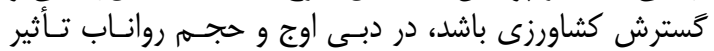

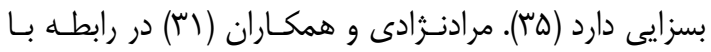

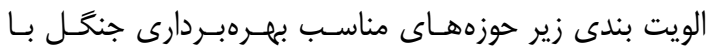

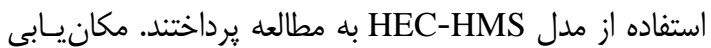

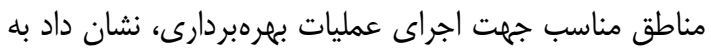

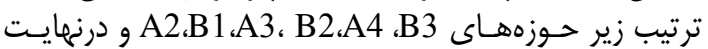

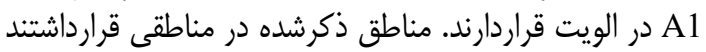

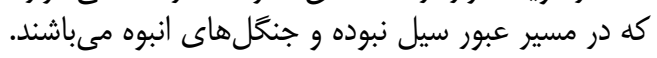

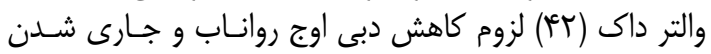

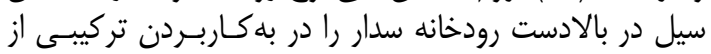

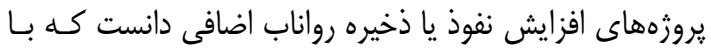

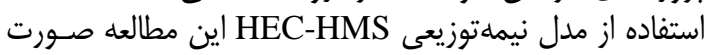

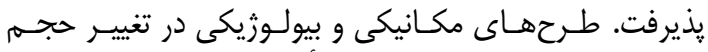

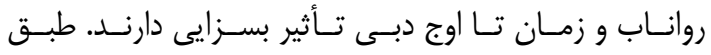

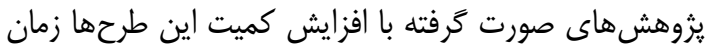

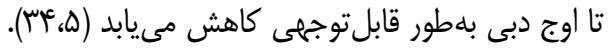

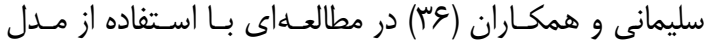

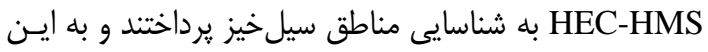

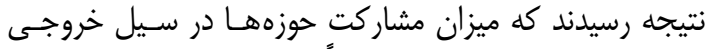

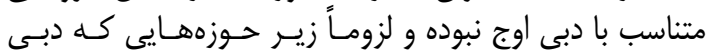

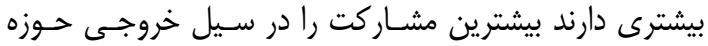

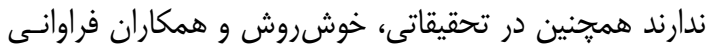

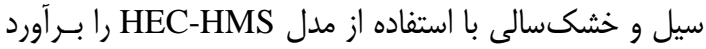
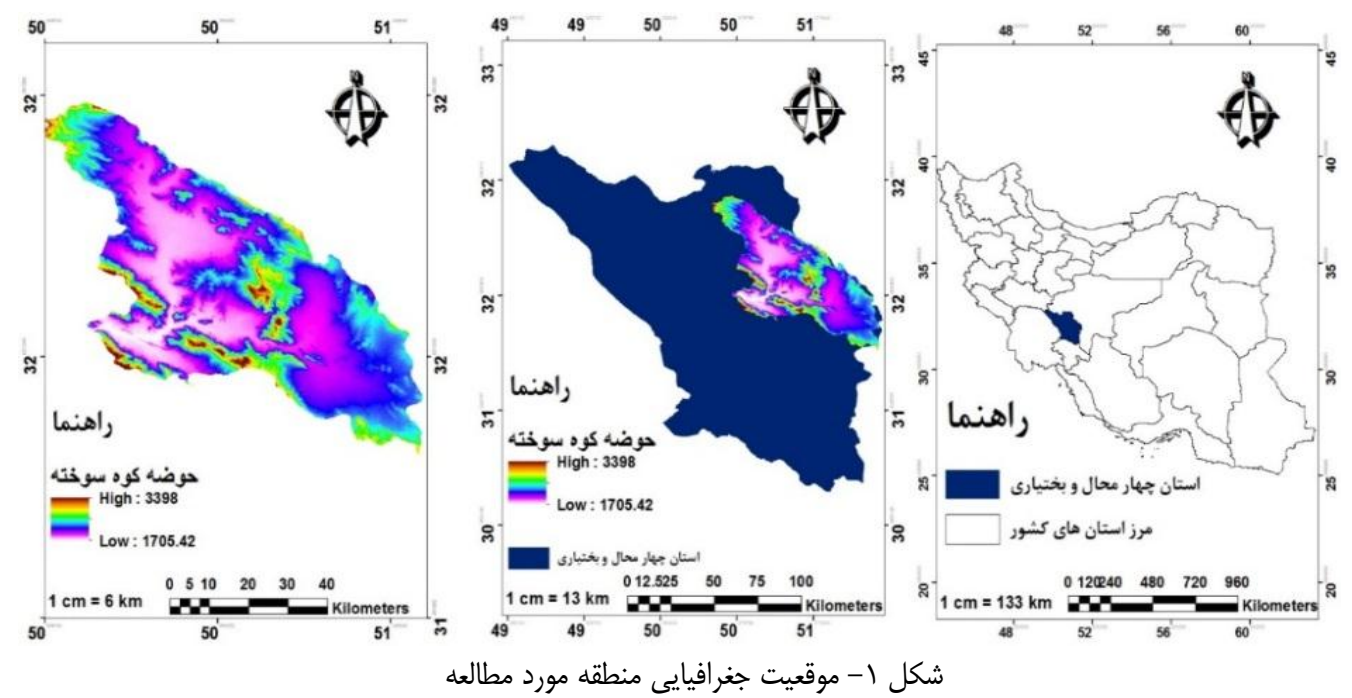

Figure 1. Location of Study area 


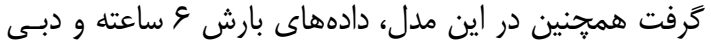

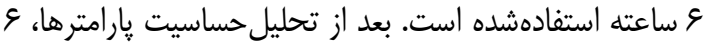

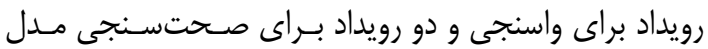

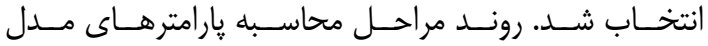
مل مل به شرح ذيل است:

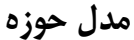

در اين قسمت بهمنظور محاسبه يارامترهـاى موردنيـاز در

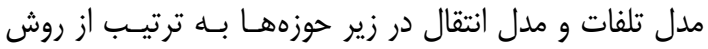

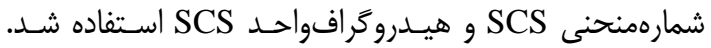

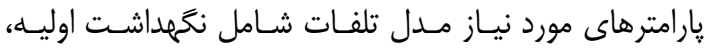
شمارهمنحنى و نفوذنايـــيرى اسـت و وــارامتر موردنيـاز مــلـل كرديد.

$T_{c}=\frac{0.96 L^{1.2}}{H^{0.2} A^{0.1}}$

$T_{\text {lag }}=0.6 T c$

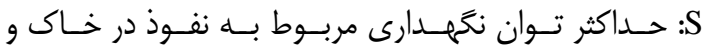

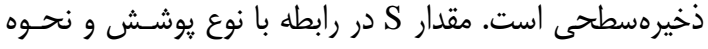

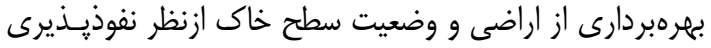

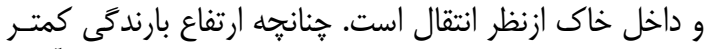

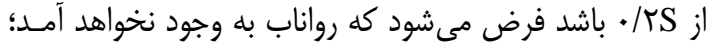

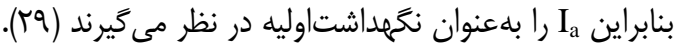

$$
\begin{aligned}
& I_{a}=0.2 S \\
& S=\frac{25400-254 C N}{C N}
\end{aligned}
$$

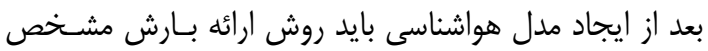

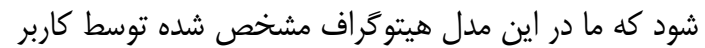

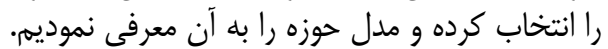

تحليل حساسيت

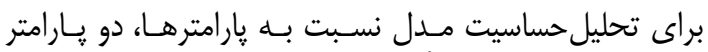

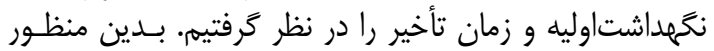

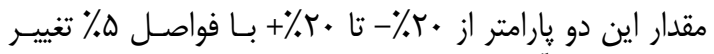

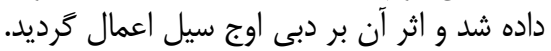

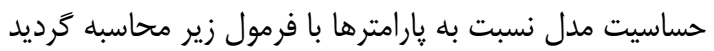

$$
\text { SEN }=100 \frac{\mathrm{New}-\text { Old }}{\text { Old. }|P C|}
$$

با توجه به اينكه دقت بارامترهاى ورودى كليد موفقيت در

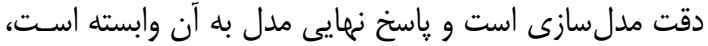

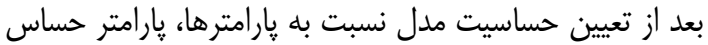

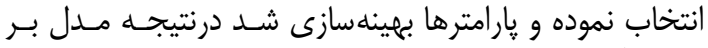
اساس آنها واسنجى كرديد.
روش كار

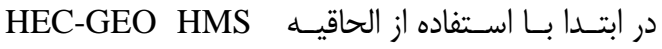

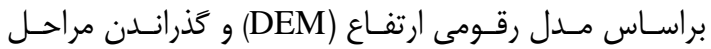

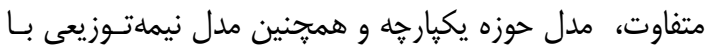

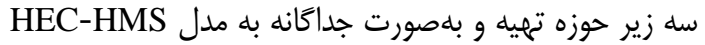

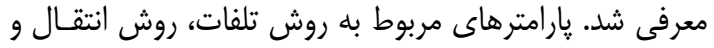

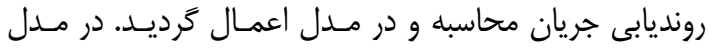

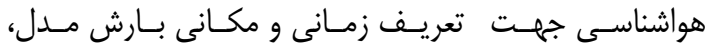

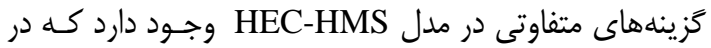

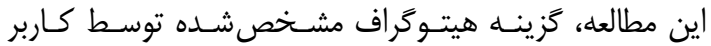

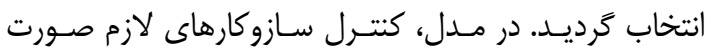
انتقال، زمان تأخير است. با اسـتفاده از روش برانسبـى - ويليـامز زمـانتـأخير محاسـبه

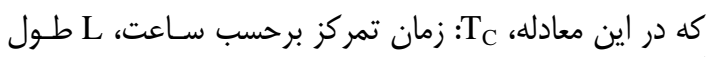

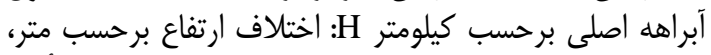

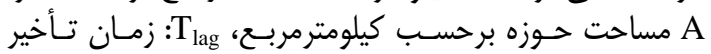
بر بارب ساعت است.

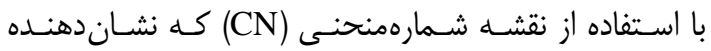

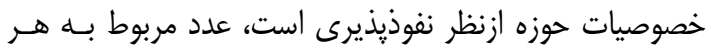
زير حوزه بلهصورت ميانگين وزنى محاسبه شد.

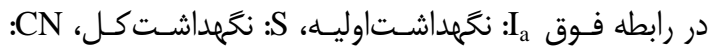
شمارهمنحنى است.

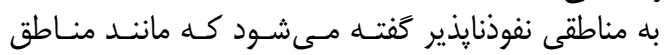

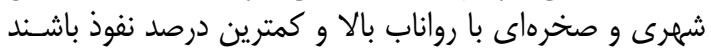

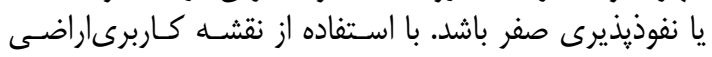

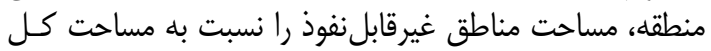

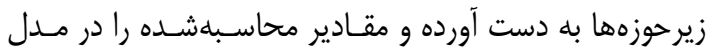

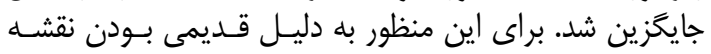

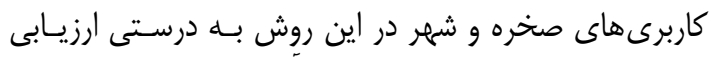

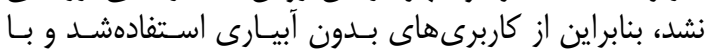

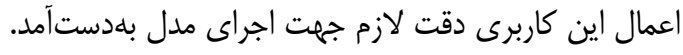

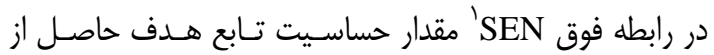

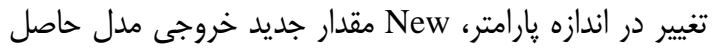

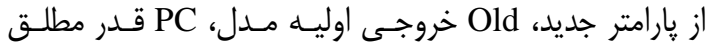
درصد تغيرات است. 


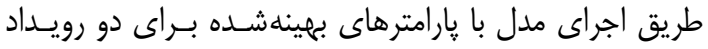

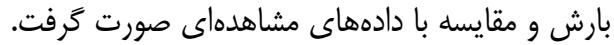

ارزيابى صحت دادهها

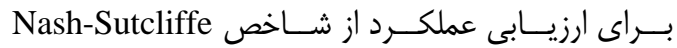

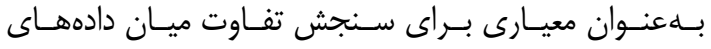

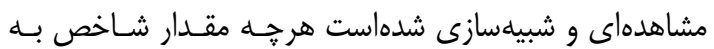

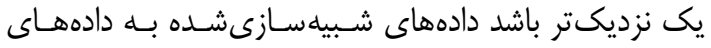
مشاهدهاى شباهت بيشترى دارند.

$$
N S E=1-\frac{\sum_{i=1}^{n}\left(O_{i-} P_{i}\right)^{2}}{\sum_{i=1}^{n}\left(O_{i-} O^{-}\right)^{2}}
$$

كوجگ در يارامتر مـوردنظر باعـث تغييـرات زيـادى در مــدل

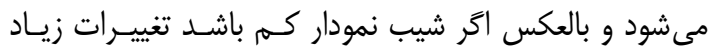

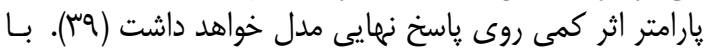

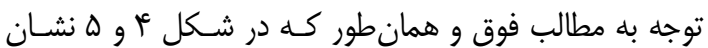

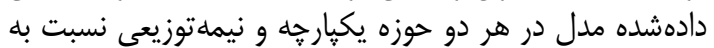

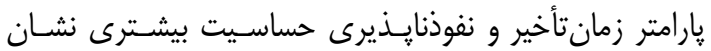

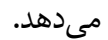

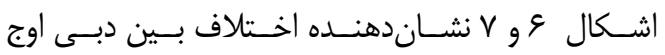

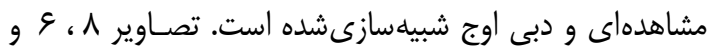

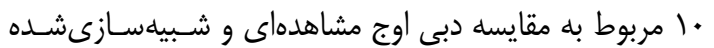

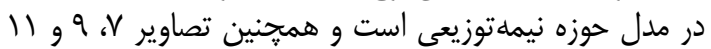

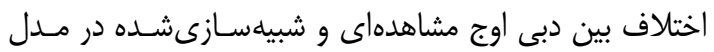

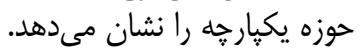

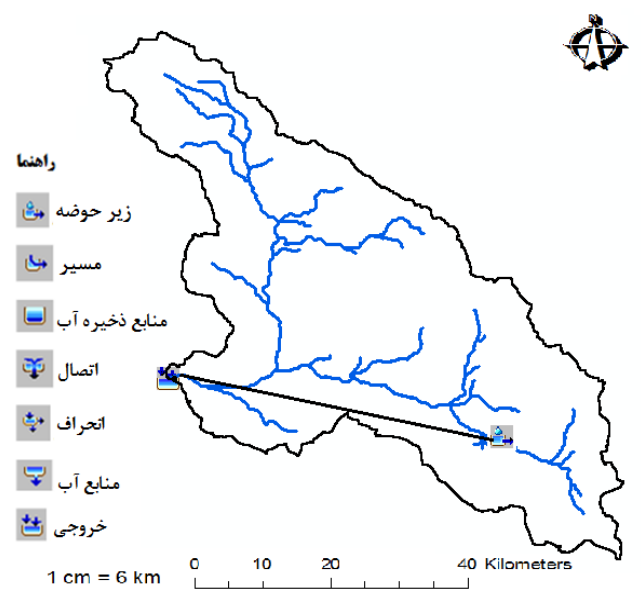

شكل س- نقشه حوزه يكيارتجه كوهسوخته بهمنظور ورود به مدل HEC-HMS

Figure 3.The Lumped watershed map of Kohsukhteh for enter to the HEC-HMS model
در اين يزوهش از 9 رويداد بارش براى واسنجى مدل استفاده

\section{شد. - مرن}

صحتسنجى

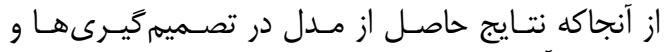

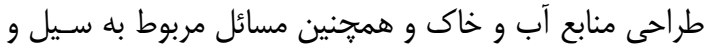

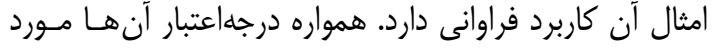

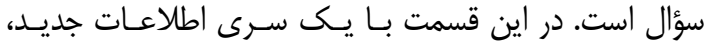

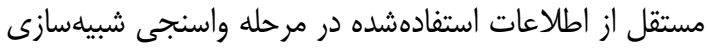

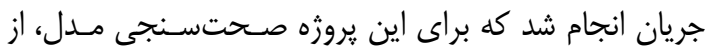

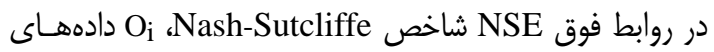
مشاهدهاى، مشاده

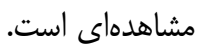

\section{نتايج و بحث مائ}

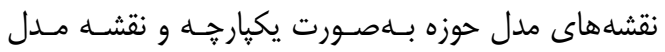

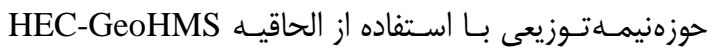

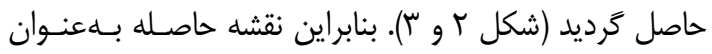

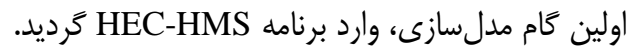

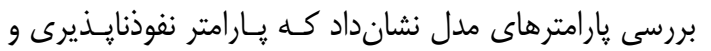

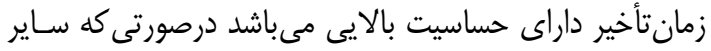

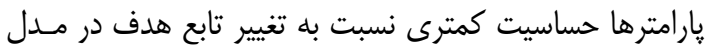
HEC-HMS باشد حساسيت مدل نسبت به يارامتر بيشتر است و يكى تغيك نغييـر

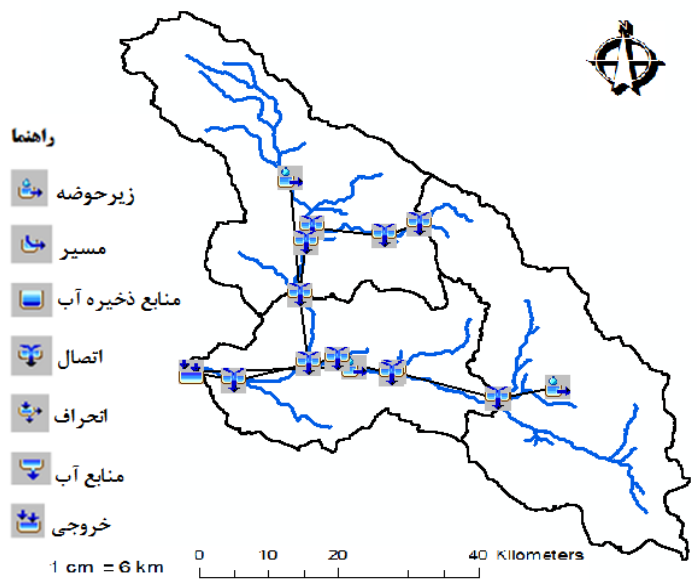

شكل r- نقشه حوزه نيمهتوزيعي كوهسوخته بdمنظور ورود به مدل

Figure 2. The semi-distributive watershed map of Kohsukhteh for enter to the HEC-HMS model 


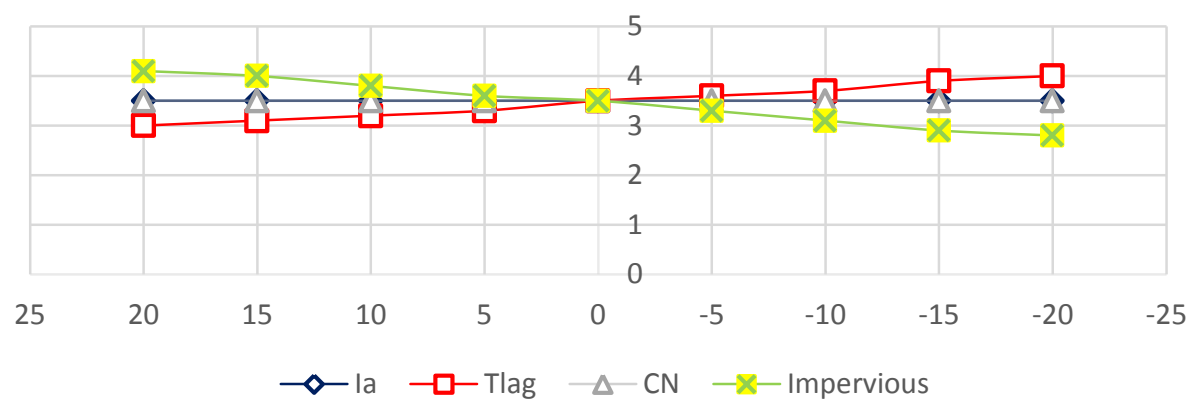

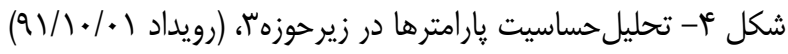

Figure 4. Analysis of the sensitivity of parameters in sub-catchment 3 (event 2012/12/21)

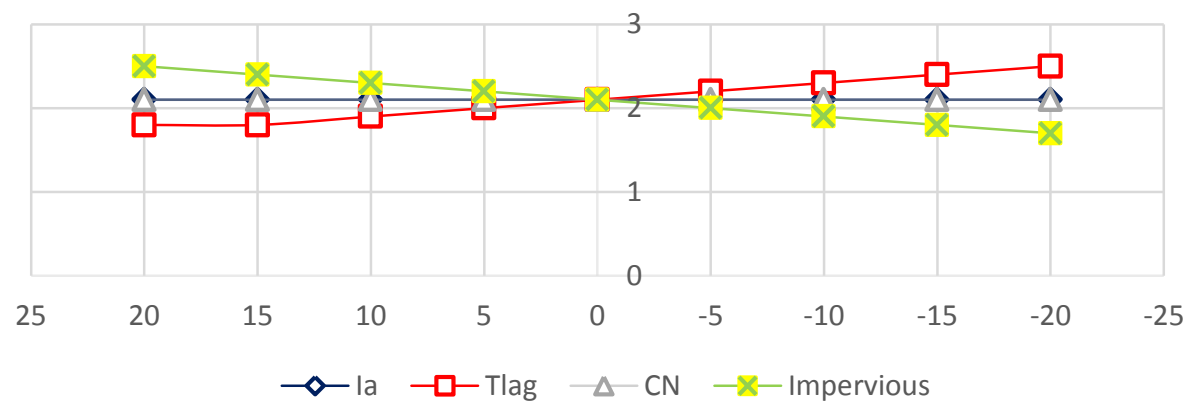

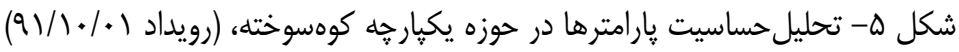

Figure 5. Analysis of the sensitivity of parameters in the Lumped Kohsukhteh watershed (event 2012/12/21) 


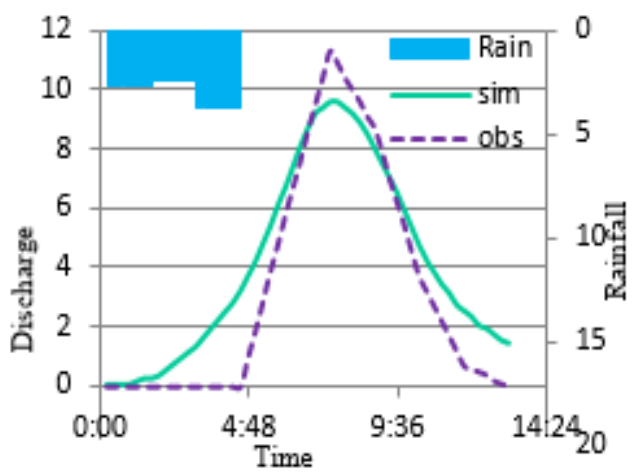

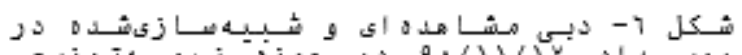

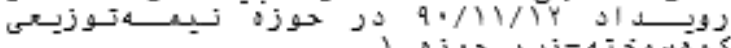
Fig. 6. Ubservational and simulated discharge in the event of $2012 / 2 / 1 \mathrm{~m}$ the sem1-distrnbutive Kohsulkhteh watershed-sub-catchmentl

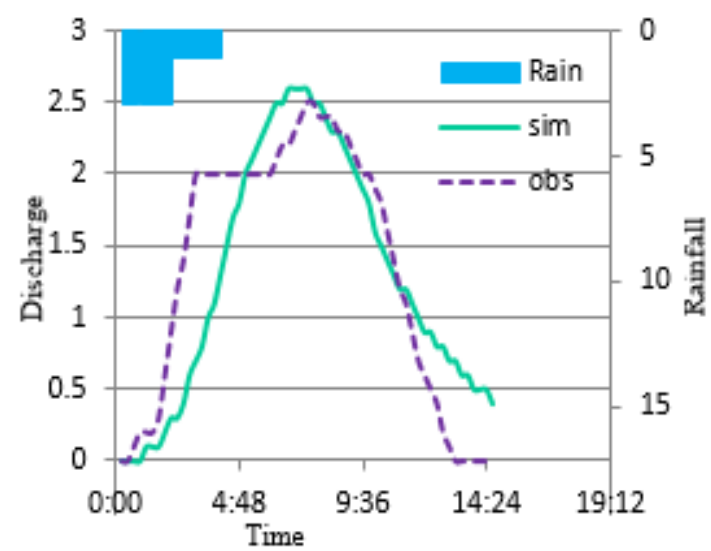

روبكل كورة Fig. 8. Ubservational and simulated discharge in the event of $2012 / 12 / 11 \mathrm{~m}$ the sem1-distributive Kohsukhteh watershed-sub-catchment 2

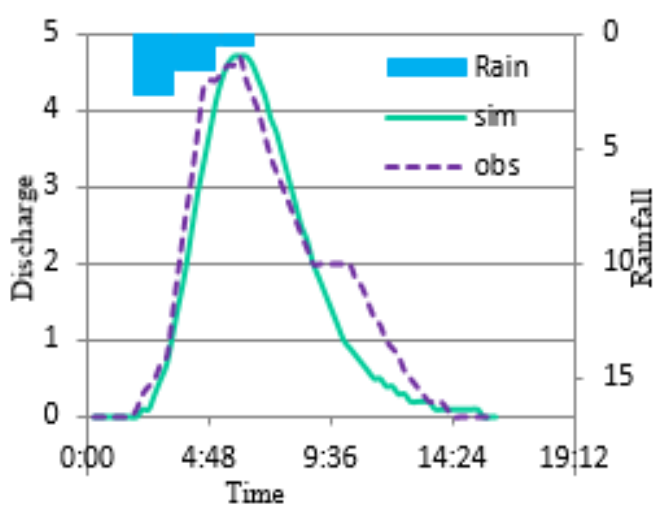

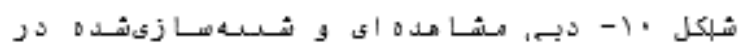

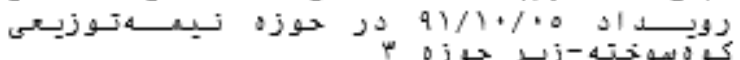
Fig. 10. Ubservational and simulated discharge in the event of $2012 / 12 / 25$ in the sem1-distributive Kohsukhteh watershed-sub-catchment 3

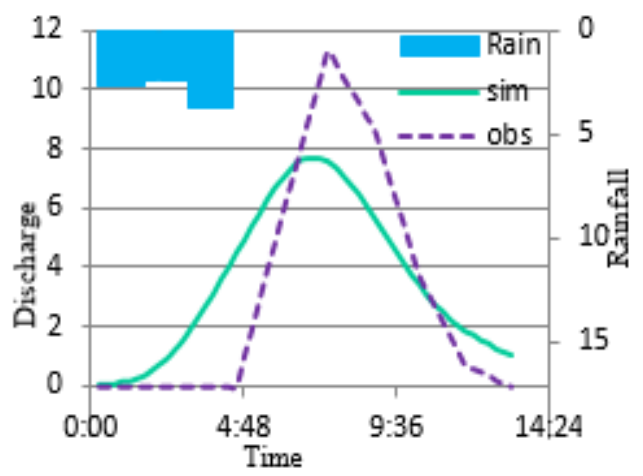

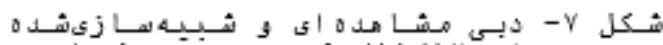

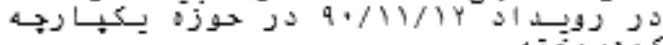
Fig. /. Ubservational and simulated discharge in the event of $2012 / 2 / 1$ in the Lumped Kohsulkhteh watershed.

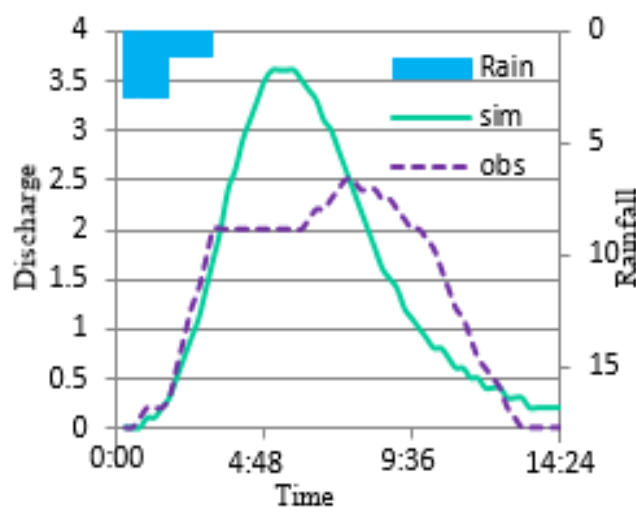

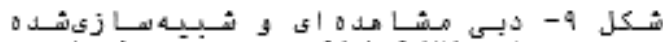

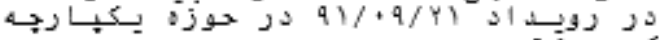

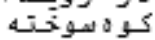
Fig. 9 . Ubservational and simulated discharge in the event of 2012/12/11 in the Lumped Kohsukhteh watershed.

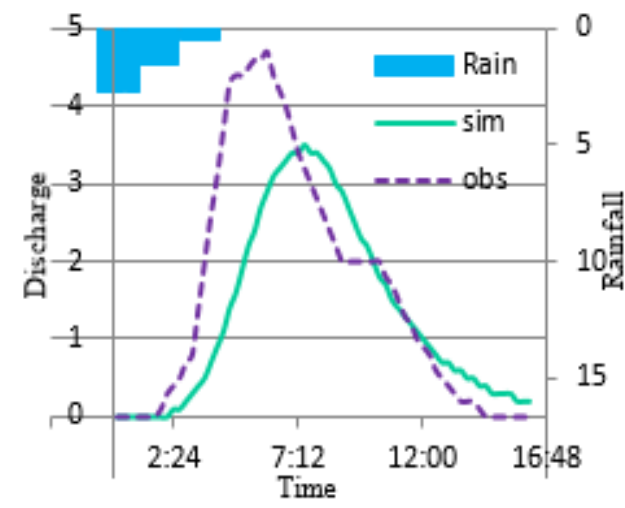

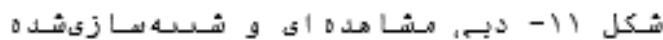

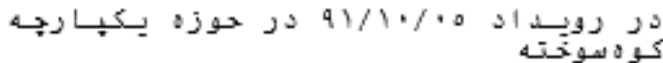

Fig. 11. Ubservational and simulated discharge in the event of $2012 / 12 / 25 \mathrm{~m}$ the Lumped Kohsulkteh watershed. 
نفوذنايذيرى و شماره منحنى واسنجى كرديد. نتايج حاصل از از واسنجى مطابق جدول (1) به دست آمد.

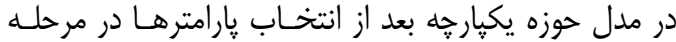

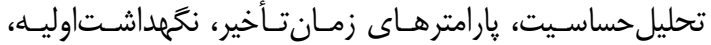

جدول (- يار امترهاى واسنجى شده در حوزه يكيارجه كوهسوخته

Table 1. Calibrated parameters in the lumped watersheh Kohsukhteh watershed

\begin{tabular}{|c|c|c|c|c|c|}
\hline حوزه & مساحت (km²) & شمارهمنحنى (CN) & نخهراشت اوليه (mm) & زمان تأخير (min) & نفوذنايذيرى (\%) \\
\hline كوهسوخته & TVAr & $V / 9 \Lambda$ & $T V / \&$ & $\Delta M N / q$ & $T / 1$ \\
\hline
\end{tabular}

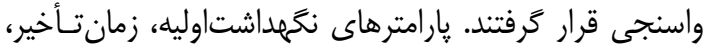

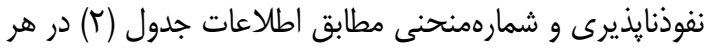

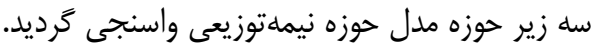

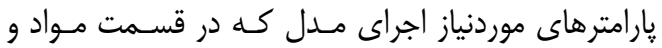

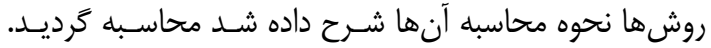

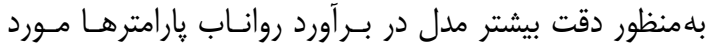

جدول r- يارامترهاى واسنجى شده در حوزه نيماتوزيعى كوهسوخته

Table 2. Calibrated parameters in the semi-distributive Kohsukhteh watersheh

\begin{tabular}{|c|c|c|c|c|c|}
\hline زير حوزه & مساحت (km²) & شمارهمنحنى (CN) & نكمبداشت اوليه (mm) & نفوذنايذيرى (\%) & زمان تأخير (min) \\
\hline 1 & $1 . \cdot V / \Delta r$ & 99 & a. & $1 / \Gamma \Delta$ & $1 \cdots N / \Delta$ \\
\hline$r$ & $V V^{\leftarrow} / / \Delta D$ & $\Delta \Lambda$ & $\Delta$. & $r$ & $\cdots \Delta / V$ \\
\hline r & $1 . \mu r / q r$ & 98 & ఎ. & $1 / 4$ & $981.5 \%$ \\
\hline
\end{tabular}

نيمهتوزيعى در مراحل واسـنجى و صـحتسـنجى مطـابق بـاــا جدول (أ) مورد مقايسه قرار كرفت.

نتايج حاصل از واسنجى در و رويداد و نتايج صحتسنجى (بر

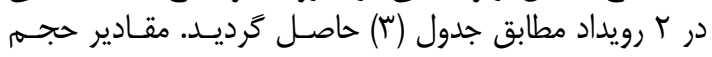

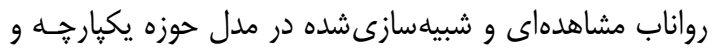

جدول س- نتايج واسنجى و صحتسنجى حوزه يكيار خه و نيمهتوزيعى كوهسوخته

Table 3. Calibration and validation results of the lumped and semi-distributive Kohsukhteh watersheh

\begin{tabular}{|c|c|c|c|c|}
\hline \multirow{3}{*}{ مرحله } & \multirow{3}{*}{ تاريخ } & \multicolumn{2}{|c|}{ شبيهسازى } & \multirow{2}{*}{ مشم| مشاهدهاى } \\
\hline & & \multicolumn{2}{|c|}{ حجم| $\left(\mathrm{m}^{3}\right.$} & \\
\hline & & نيماتوزيعى & يكيار جه & \\
\hline \multirow{6}{*}{ واسنجى } & $1 r q . / \cdot \Lambda / r q$ & $\cdot / \Lambda \mid$ &.$/ 9$ &.$/ 90$ \\
\hline & $1 r q . / 1 / / r$ & $1 / r$ & $1 / \Lambda$ & $1 / 1$ \\
\hline & $\mid$ |rq $/ \cdot 1 / 11$ &.$/ 4 V$ & سז/. &.$/ \mu$ \\
\hline & $|r q| / \cdot q / r \mid$ & q & $.1 \Delta \omega$ &.$/ 4$ \\
\hline & $1+99 / 1.1 .1$ & . & זr/. ( l &.$/ 4$ \\
\hline & $\mid(\% q \mid / 1 . / .0$ &.$/ T \Delta$ &.$/ 4$ &.$/ T \Delta$ \\
\hline \multirow[b]{2}{*}{ صحتسنجى } & $1 r q \mid / 1 r / 1 \Lambda$ & $1 / 1$ &.$/ 9$ & $1 / r$ \\
\hline & וrq &.$|9|$ &.$/ \% \omega$ &.$/ 8$ \\
\hline
\end{tabular}

جدول ع- ارزيابى نتايج مدلهاى نيمهتوزيعى و يكيار خه حوزه كوهسوخته Table 4. Evaluation of the results of semi-distributive and lumped models of Kohsukhteh watersheh

\begin{tabular}{|c|c|c|c|c|}
\hline \multirow[b]{2}{*}{ حوزه } & \multirow{2}{*}{ مرحله } & \multirow{2}{*}{ رويداد } & \multicolumn{2}{|c|}{ NAS } \\
\hline & & & مدل يكهار & مدل نيمهتوزيعى \\
\hline \multirow{8}{*}{ كوهسوخته } & \multirow{6}{*}{ واسنجى } & $1 r q . / \cdot N / r q$ & $\cdot / \mu \mid$ & $\cdot / 1$ \\
\hline & & וrq./11/ & $\cdot / \uparrow \wedge$ & - \\
\hline & & $|r q| / \cdot 1 / 11$ & rar & $\cdot / \mathrm{V}$ \\
\hline & & $|r q| / \cdot q / r \mid$ & . &.$/ M$ \\
\hline & & $|r q| / 1 . / .1$ &.$/ 9$ &.$/ 91$ \\
\hline & & $|r q| / 1 . / .0$ & .190 &.$/ 94$ \\
\hline & \multirow[t]{2}{*}{ صحتسنجى } & $|r q| / \mid r / / q$ & $\cdot / \Delta$ & $\cdot / 1$ \\
\hline & & $\mid r q T / \cdot 1 / T^{c}$ &. $\mid 94$ & - / A \\
\hline
\end{tabular}

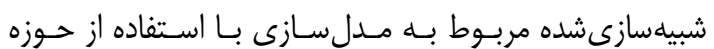

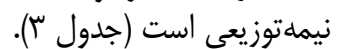

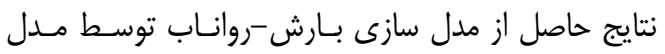

HEC-HMS

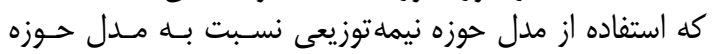

نتايج حاصل از واسنجى و بهينه سـازى نشـان داد كـهـ در

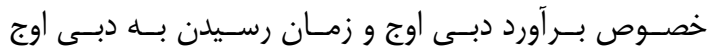

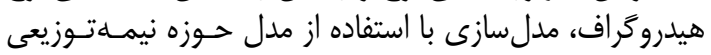

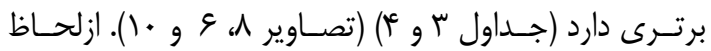

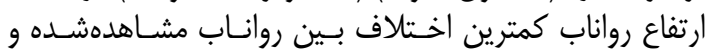




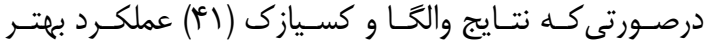

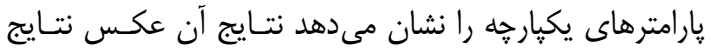

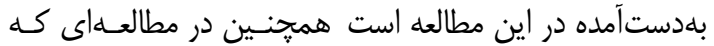

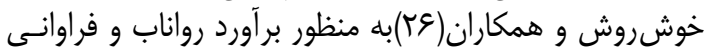

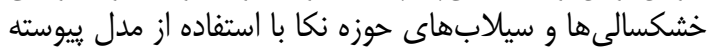

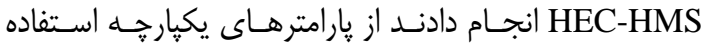

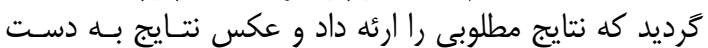
مده در مطالعه حاضر است.

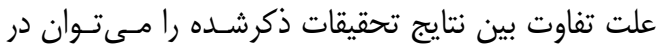

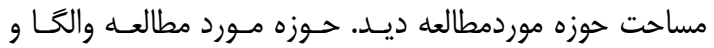

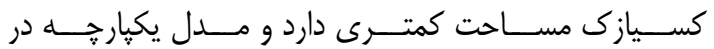

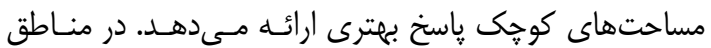

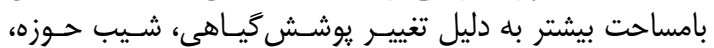

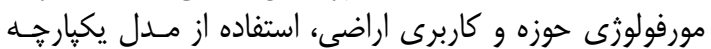

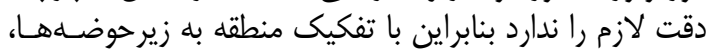

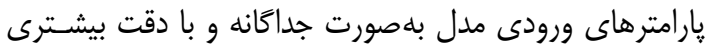

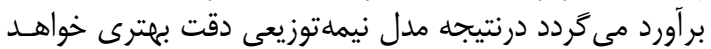

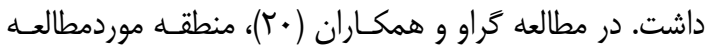

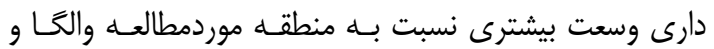

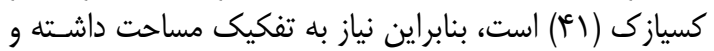

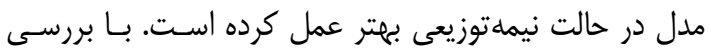

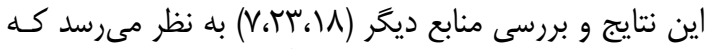

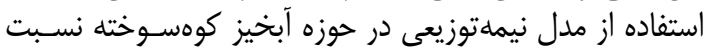

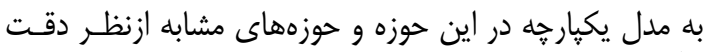
برآورد رواناب، برترى دارد.

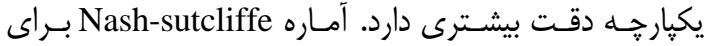

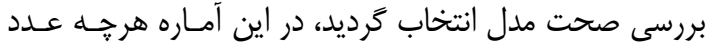

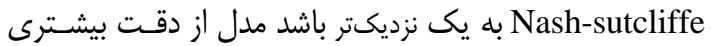

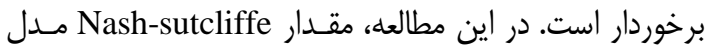

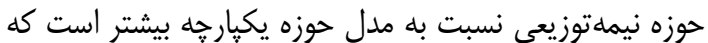

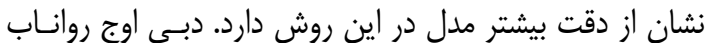

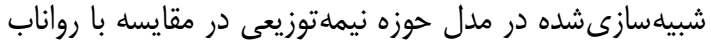

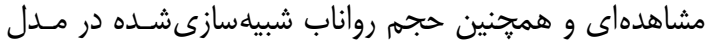

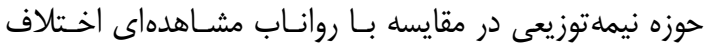

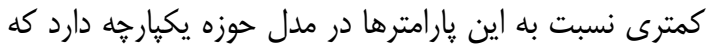

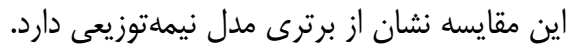

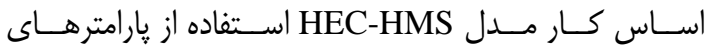

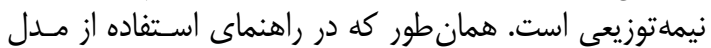

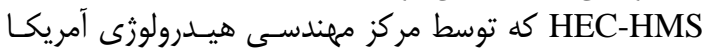

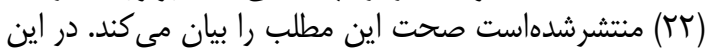

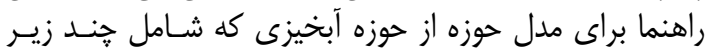

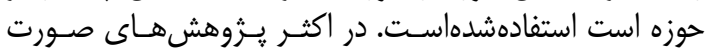

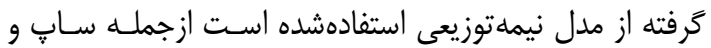

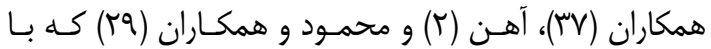

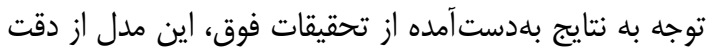

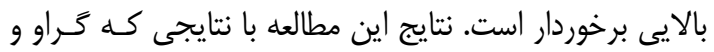

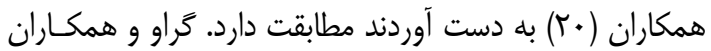

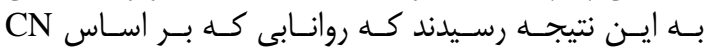

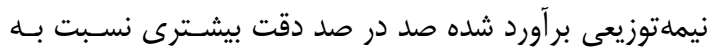

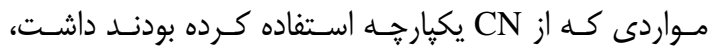

1. Adamowski, J. 2013. Using support vector regression to predict direct runoff base fl ow and tot ow in a mountainous watershed with limited data in Uttaranchal, India. Annals of Warsaw University of Life Sciences-SGGW. Land Reclamation, 45(1): 71-83.

2. Ahn, G. 2007. The effect of urbanization on the hydrologic regim of the bigdarby creek watershed, Ohio. (PHD) Thesis, Ohio State University, Ohio,USA, 189 pp.

3. Agrawal, A. 2005. A data model with pre-and-post processor for HEC-HMS. (MSc) Thesis, Texas A\&M University, Texas, USA, $210 \mathrm{pp}$.

4. Alexander Braud, J. 2009. Impact of watershed delineation detail on hydrologic process modeling in low slop areas. M.Sc. Thesis, Louisiana State University, Louisiana, USA. 123 pp.

5. Ariapour, A. and K. Karami. 2013. Anticipated effect of change in rangland in amount runoff using HEC-HMS in Golrod Boroujerd watershed. Journal of Iran's Natural Ecosystems, 9: 97-115 (In Persian).

6. Asadi, A. and F. Boustani. 2013. Performance evaluation of the HEC-HMS hydrologic model for lumped and semi-distributed stormflow simulation (StudyArea: Delibajak Watershad). American Journal of Engineering Research (AJER), 2(11): 115-121.

7. Azagra E. 1998. Rainfall runoff in the Guadalupe River Basin. CE 397 GIS in Water Resources.

8. Bardossy, A. and T. Das. 2008. Rainfall network on model calibration and application. Hydrology and Earth System Sciences Discussions, 12: 77-89.

9. Behnam, P., M. Sayannegad and A. Ebrahimi. 2012. The effect of land use on flood hydrograph of Zayanderod river in Esfahan metropolitan area. Journal Water and Wastewater, 4: 103-111 (In Persian)

10. Bendient, P.B., W.C. Huber and B.E. Vieux. 2013. Hydrology and floodplain analysis. Pearson, england.780,297-339pp.

11. Beven, K. 2012. Rainfall-Runoff Modeling: The Primer. John Wiley \& Sons.450,51-79pp.

12. Bhadra, A., N. Panigrahy, R. Singh, N.S. Raghuwanshi, B.C. Mal and M.P. Tripathi. 2008. Development of a geomorphological instantaneous unit hydrograph model for scantily gauged watersheds. Journal of Environmental Modelling and Software, 23: 1013-1025.

13. Boggs, J.L. and G. Sun. 2011. Urbanization alters watershed hydrology in the Piedmont of North Carolina. Ecohydrology, 4(2): 256-264.

14. Booij, M.J. 2003. Determination and integration of appropriate spatial scales for river basin modeling. Hydrol Process, 17: 2581-2598. 
15. Bormann, H. 2006. Impact of spatial data resolution on simulated catchment water balances and model performance of the multiscale TOPLATS model. Hydrology and earth System Science, 10: $165-179$.

16. Butts, M.B., J.T. Payne, M. Kristensen and H. Madsen. 2004. An evaluation of the impact of model structure on hydrological modeling uncertainty for streamfl ow prediction. Journal of Hydrology, 298: 242-266.

17. Cabral Moreira, A.A. 2013. Modelagem hidrologica da bacia hidrografica do Rio Granjeiro- CratoCE. (MSc) Thesis, Universidade Federal do Ceara, 164 pp.

18. Chu, X., A.M. ASCE and A. Steinman. 2009. Event and Continuous Hydrologic Modeling with HECHMS. Journal of Irrigation and Drainage Engineering, 135: 119-125.

19. Elbialy, S., A. Mahmoud, B. Pradhan and M. Buchroithner. 2013. Application of spaceborne synthetic aperture radar data for exteaction soil moisture and its use in hyrological modeling at gottleuba. Journal of Flood rick management, 7(2): 159-175.

20. Grove, M., J. Harbor and B. Engle. 1998. Composite vs. distributed curve numbers:effects on estimates of storm runoff depths. JAWRA Journal of the American Water Resources Association, 34(5):1015-1023.

21. Ibrahim-Bathis. K. and S.A. Ahmed. 2016. Rainfall-runoff modelling of Doddahalla watershed. Arabian Journal of Geosciences, 9(3): 170.

22. Hydrologic Engineering Center- Hydrologic Modelig System. 2013. Hydrologic Engineering CenterHydrologic Modelig System (HEC-HMS) Users manual (Version 4.0). US Army Crops of Engineers.

23. Kabiri, R., V. Ramani Bai and A. Chan. 2015. Assessment of hvdrologic impacts of climate change on the runoff trend in Klang Watershed, Malaysia. Environmental Earth Sciences, 73: 27-37.

24. Karabowa, B., A.E. Sikorska, K. Banasik and S. Kohnova. 2012. Parameters determination of a conceptual rainfall-runoff model for a small catchment in Carpathians. Annals of Warsaw University of Life Sciences-SGGW. Land Reclamation, 44(2): 155-162.

25. Karimi, M., H. Maleki Nejad, A. Abghari and M.S. Aziziyan. 2011. Assess different methods simulation of flood hydrograph using HEC-HMS package (Case Study: Chehelgazi Watershed), Journal of Iran Water Research, 9: 29-38 (In Persian).

26. Khoshravesh, M., M. Raeeni and E. Nikzad Tehrani. 2016. Application Continuous rainfall-runoff models HMS-SMA And Freauencv Droughts and floods in Neka Watershed under climate scenarios $\mathrm{A}_{2}$ Model HadCM3, Journal of Watershed Management Research , 7(14): 128-140 (In Persian).

27. Lu, H., T. Hou, R. Horton, Y. Zhu, X. Chen, Y. Jia, W. Wang and X. Fu. 2013. The stream flow estimation using the xinanjiang rainfall runoff model and dual state-parameter estimation method. Journal of Hydrology, 480: 102-114.

28. Mahdavi, M. 2007. Applied Hydrology, Tehran University Press, 425 (2):135-145

29. Mahmood, R., S. Jia and M.S. Babel. 2016. Potential impacts of climate change on water resources in the Kunhar river basin, Pakistan. Water, 8(1): 2-24.

30. Meenu, R., S. Rehana and P.P. Muju. 2013. Assesment of hydrological imnacts of change in TungaBhadra river basin India with HEC-HMS and SDSM. Journal of Hydrological Processes, 27(11): 1572-1589.

31. Moradnejadi, M., M. Jor Gholami and A. Malekian. 2015. Sub-basins prioritize the right to exploit the forest using HEC-HMS model (Case Study: Kheirood forest), Forest and wood products, Iranian Journal of Natural Resources, 2: 405-418 (In Persian).

32. Nadala, H. and U.R. Ratanayke. 2011. Flood risk analysis using Fuzzy models. Journal of Flood risk management, 2: 121-128.

33. Noori, N., L. Kalin, S. Sen, P. Srivastava and C. Lebleu. 2016. Identifying areas sensitive to land use/land cover changefor downstream flooding in a coastal Alabama watershed. Regional Environmental change, 16(6): 1833-1845.

34. Norali, M. and B. Ghahreman. 2017. Assessing the impact of watershed management operation on flood hvdrograph model HEC-HMS (Case Study: Watershed Gosh and Bahre), Journal of Watershed Management Research, 7(13): 60-71 (In Persian).

35. Olang, L.O. and J. Furst. 2011. Effects of land cover change on flood peak discharges and runoff volumes: model estimates for the Nyando River Basin, Kenya. Hydrological Processes, 25(1): 80-89.

36. Soleimani, K., M.B. Gonbad, S.R. Mousavi, Sh. Khaliq. 2008. The potential for flooding in catchments using HEC-HMS model in GIS (Case Study kasilian catchment). Physical Geography Researches, 65: 51-60 (In Persian).

37. Sup, M.S., S.M. Taley and M.U. Kale. 2015. Rainfall-runoff modeling using HEC-HMS for Wan river basin. Internatinal Journal of Research in Engineering, Science and Technologies, 28: 21-30.

38. Szalinska, W. and T. Zawislak. 2005. The use of radar data for spatial interpolation ofprecipitation. In: Hydrology, meteorology, climatology- research and forecasts in the era of computerization. Institute of Meteorology and water Management, Monograph, 195-205.

39. Telveri, A. 1996. Hydrologic models in a simple word, The Research institute of forests and Rangelands, (In Persian).

40. Vassova, D. 2013. Comparison of rainfall-runoff models for design discharge. Soil and Water Research, 8(1): 26-33.

41. Walega, A. and L. Ksiazek. 2015. The effect of a hydrological model structure and rainfall data. Annals of Warsaw University of Life Sciences-SGGW, 47(4): 305-321.

42. Walter Drake, Chad. 2014. Assesment of flood Mitigation Strategies For Reducing Peak Discharges in The Upper Cedar River Watershed. (M.Sc.) Thesis, University of Iowa, Iowa. 229 pp.

43. Zhang, C., J. Chu and G. fu. 2013. Sobol's sensitivity analysis for a distributed hydrological model of Yichun River Basin. China Journal of Hydrology, 480: 58-68. 


\title{
Assessment Performance of HEC-HMS Hydrological Model for the Lumped and Semi-distributive Watershed (Case Study: Kohsukhteh Catchment)
}

\author{
Sodabeh Behyan Motlagh ${ }^{1}$, Mehdi Pajoohesh ${ }^{2}$, Afshin Honarbakhsh $^{3}$ and \\ Negar Salehi Hafshejani ${ }^{4}$
}

1- M.Sc. Student, Shahrekord University (Corresponding author: sodabehbehyan@gmail.com) 2 and3- Assistant Professor and Associate Professor, Shahrekord University 4- Graduate Master Malayer University

Received: May 5, $2016 \quad$ Accepted: September 5, 2017

\begin{abstract}
For modeling, the concept of the system and the system boundary is necessary. The system is defined as a group of objects that in order to fulfill a specific purpose in the framework relationship or interdependence of regularly are interconnected. Systems rainfall - runoff from rainfall in the basin is started and after applying the types of losses (evaporation, infiltration, etc) it will become runoff. In the study of the HEC-HMS model for show the effectiveness of the sub-basin in runoff of the watershed is used; so SCS curve number method for losses method and SCS unit hydrograph method for transmission method were used. In beginning distribution basin model with three sub-basin then as an lumped basin model was run. The results show that the accuracy of the model in the watershed by taking sub-basin is more than lumped basin model.
\end{abstract}

Keywords: Lumped basin, Semi Distribution basin, Hydrologic model, SCS 